INPLASY

PROTOCOL

To cite: Su et al. Efficacy of acupuncture in promoting postoperative recovery from rotator cuff injuries: systemic Evaluation and meta-analysis. Inplasy protocol 202220115. doi:

10.37766/inplasy2022.2.0115

Received: 26 February 2022

Published: 26 February 2022

Corresponding author:

Hui Su

suhui1334676310@163.com

Author Affiliation:

Shandong University of

Traditional Chinese Medicine.

Support: National Natural

Science Found.

Review Stage at time of this submission: Preliminary searches.

Conflicts of interest:

None declared.

\section{Efficacy of acupuncture in promoting postoperative recovery from rotator cuff injuries: systemic Evaluation and meta-analysis}

\author{
Su, H1; Gao, S2; Wang, R33 Yan, B4; Tan, G5; Xu, Z6.
}

Review question / Objective: In previous systematic reviews of studies, there were limited studies on the clinical efficacy of acupuncture after rotator cuff injury and lacked strong convincing. Therefore, we decided to conduct a systematic review based on the best evidence and methods currently available to assess the effectiveness and safety of acupuncture for postoperative pain and function of rotator cuff injuries.

Condition being studied: Efficacy of acupuncture in promoting postoperative recovery from rotator cuff injuries.

INPLASY registration number: This protocol was registered with the International Platform of Registered Systematic Review and Meta-Analysis Protocols (INPLASY) on 26 February 2022 and was last updated on 26 February 2022 (registration number INPLASY202220115).

\section{INTRODUCTION}

Review question / Objective: $\mathbf{n}$ previous systematic reviews of studies, there were limited studies on the clinical efficacy of acupuncture after rotator cuff injury and lacked strong convincing. Therefore, we decided to conduct a systematic review based on the best evidence and methods currently available to assess the effectiveness and safety of acupuncture for 
postoperative pain and function of rotator cuff injuries.

Condition being studied: Efficacy of acupuncture in promoting postoperative recovery from rotator cuff injuries.

\section{METHODS}

Search strategy: The literature search consisted of 3 English databases (Pubmed, Cochrane, Web of sci) and 3 Chinese databases (CNKI, Wanfang, Vip). The search time is set from the establishment of each database to November 30, 2021. The search was carried out independently by 2 researchers.

Participant or population: Participants were patients clinically diagnosed with rotator cuff injury and undergoing shoulder surgery, regardless of gender and origin.

Intervention: All randomized controlled comparisons of acupuncture and other forms of controlled modalities. Acupuncture treatment was defined as manual acupuncture, electro-acupuncture and warm acupuncture.

Comparator: The control group was conventional physical exercise treatment.

\section{Study designs to be included: RCT.}

Eligibility criteria: The inclusion criteria for inclusion and exclusion were: (I)participants were patients clinically diagnosed with rotator cuff injury and undergoing shoulder surgery, regardless of gender and origin; (II) all were RCT studies; (III) various forms of acupuncture treatment including acupuncture in the experimental group; (iv) participants were included in the criteria, diagnostic criteria, and efficacy evaluation criteria were clear; (v) evaluation indicators including clinical treatment efficiency, visual simulation scale (VAS) score, Constant-Murley score (CMS) as well as the UCLA shoulder score. Exclusion criteria are as follows: (I) non-randomized controlled trials such as retrospective analyses, case reports, cohort studies, etc.; (II) unpublished literature; (III) participants undergoing surgical treatment (iv) literature on animals.

Information sources: The literature search consisted of 3 English databases (Pubmed, Cochrane, Web of sci) and 3 Chinese databases (CNKI, Wanfang, Vip).

Main outcome(s): Clinically effective. The effective and significant effective rates in the literature were uniformly classified as clinical effective rates.Pain intensity: measured by validated scales, (VAS) the visual analog scale ) Shoulder function: measured by validated scales, the Constant-Murley score, (UCL) University of California-Los Angeles Shoulder rating scale).

Quality assessment / Risk of bias analysis: The Cochrane Collaboration tool was used to assess the risk of bias of the selected studies.

Strategy of data synthesis: The Cochrane Collaboration tool was used to assess the risk of bias of the selected studies Shamseer L, Moher D, Clarke M, GThe review included whether the random method was described correctly, whether allocation concealment was used, whether the subjects were blinded, whether the outcome evaluators were blinded, whether the study data were complete, whether there were selective reports, and whether there were other biases.The following aspects were assessed independently by two reviewers, random sequence generation, allocation concealment, blinding of participants and personnel, blinding of outcome assessment, incomplete outcome data, selective reporting and other bias. Disagreements were analyzed by the third reviewer.

Subgroup analysis: No.

Sensitivity analysis: The statistical method used RevMan5.3 software (Cochrane Collaboration, Copenhagen, Denmark) to meta-analyze the data, and the continuous results were expressed as mean difference (MD) with a $95 \%$ confidence interval (CI). 
$\mathrm{p}<$ of 0.05 is considered significant. Heterogeneity is assessed using the i2 test. If the $12>$ is $50 \%$, a random effects model is used. Otherwise, a fixed-effects model is used. Evaluation publication bias is manifested in the form of funnel charts.

Country(ies) involved: China.

Keywords: acupuncture therapy; postoperative; meta-analysis; rotator cuff injuries.

Contributions of each author:

Author 1 - Hui Su.

Author 2 - Shang Gao.

Author 3 - Ruochong Wang.

Author 4 - Binghan Yan.

Author 5 - Guoqing Tan.

Author 6 - Zhanwang Xu. 